\title{
Altered Balance of Glutamatergic/GABAergic Synaptic Input and Associated Changes in Dendrite Morphology after BDNF Expression in BDNF-Deficient Hippocampal Neurons
}

\author{
B. Singh, ${ }^{1}$ C. Henneberger, ${ }^{1}$ D. Betances, ${ }^{1}$ M. A. Arevalo, ${ }^{2}$ A. Rodríguez-Tébar, ${ }^{2}$ J. C. Meier, ${ }^{1}$ and R. Grantyn ${ }^{1}$ \\ ${ }^{1}$ Developmental Physiology Group, Johannes Mueller Institute for Neurophysiology, University Medical School (Charité) of the Humboldt University, D- \\ 10117 Berlin, Germany, and 2Instituto de Neurobiología Cajal, Madrid, Spain
}

\begin{abstract}
Cultured neurons from $b d n f-/-$ mice display reduced densities of synaptic terminals, although in vivo these deficits are small or absent. Here we aimed at clarifying the local responses to postsynaptic brain-derived neurotrophic factor (BDNF). To this end, solitary enhanced green fluorescent protein (EGFP)-labeled hippocampal neurons from $b d n f-/-$ mice were compared with $b d n f-/-$ neurons after transfection with BDNF, $b d n f-/-$ neurons after transient exposure to exogenous BDNF, and $b d n f+/+$ neurons in wild-type cultures. Synapse development was evaluated on the basis of presynaptic immunofluorescence and whole-cell patch-clamp recording of miniature postsynaptic currents. It was found that neurons expressing BDNF::EGFP for at least $16 \mathrm{~h}$ attracted a larger number of synaptic terminals than BDNF-deficient control neurons. Transfected BDNF formed clusters in the vicinity of glutamatergic terminals and produced a stronger upregulation of synaptic terminal numbers than high levels of ambient BDNF. Glutamatergic and GABAergic synapses reacted differently to postsynaptic BDNF: glutamatergic input increased, whereas GABAergic input decreased. BDNF::EGFP-expressing neurons also differed from BDNF-deficient neurons in their dendrite morphology: they exhibited weaker dendrite elongation and stronger dendrite initiation. The upregulation of glutamatergic synaptic input and the BDNF-induced downregulation of GABAergic synaptic terminal numbers by postsynaptic BDNF depended on tyrosine receptor kinase B activity, as deduced from the blocking effects of K252a. The suppression of dendrite elongation was also prevented by block of tyrosine receptor kinase B but required, in addition, glutamate receptor activity. Dendritic length decreased with the number of glutamatergic contacts. These results illuminate the role of BDNF as a retrograde synaptic regulator of synapse development and the dependence of dendrite elongation on glutamatergic input.
\end{abstract}

Key words: synaptogenesis; dendrite growth; E/I balance; $b d n f$ knock-out; neuron transfection; retrograde messenger

\section{Introduction}

Brain-derived neurotrophic factor (BDNF) is implicated in a large number of mechanisms that are required to form and optimize neuronal connectivity in the brain (Bibel and Barde, 2000; Bi and Poo, 2001; Vicario-Abejon et al., 2002; Chao, 2003; Lu, 2003). A mutation of the BDNF gene may result in a failure to access the pathway of regulated release and in reduced cognitive performance (Egan et al., 2003). Recent experimental evidence suggests that the $32 \mathrm{kDa}$ protein pro-BDNF can be released from any part of a cell by a constitutively active mechanism (Lou et al., 2005). At extrasynaptic sites, it may bind to the low-affinity common neurotrophin receptor (NTR) p $75^{\mathrm{NTR}}$ that, in the presence of sortilin, promotes cell death (Teng and Hempstead, 2004);

Received Dec. 21, 2005; revised April 16, 2006; accepted April 24, 2006.

This work was supported by German Research Council Grants Ro925 and SFB515-B2 (R.G.) and "Ministerio de Ciencia y Tecnología" Grant PB08477 (A.R.-T.). B.S. was a postgraduate fellow of the Berlin Graduate School GRK238. We are grateful for many helpful discussions with our colleagues Uwe Heinemann, Sergei Kirischuk, and Randolf Menzel. The technical assistance of Ulrike Neumann and Kerstin Rückwardt is highly appreciated.

Correspondence should be addressed to Dr. Rosemarie Grantyn, Department of Sensory and Developmental Physiology, Johannes Mueller Center for Physiology, Humboldt University Medical School (Charité), Tucholskystrasse 2, D-10117 Berlin, Germany. E-mail: rosemarie.grantyn@charite.de.

DOI:10.1523/JNEUROSCI.5474-05.2006

Copyright $\odot 2006$ Society for Neuroscience $\quad$ 0270-6474/06/267189-12\$15.00/0 however, if sorted appropriately (Chen et al., 2005) and concentrated in the vicinity of depolarizing synapses (Hartmann et al., 2001), pro-BDNF is also released in an activity-dependent manner. After cleavage, mature BDNF ( $14 \mathrm{kDa})$ binds to presynaptic and postsynaptic tyrosine kinase $\mathrm{B}(\mathrm{TrkB})$ receptors to either activate local signaling cascades (Kalb, 2005) or exert transcriptional regulation after being transported to the soma of the presynaptic neuron (Howe and Mobley, 2005; Rind et al., 2005). Both mechanisms could account for modifications of transmitter release from axon terminals. In addition to these presynaptic changes, BDNF is reported to regulate the number and distribution of postsynaptic transmitter receptors at glutamatergic (Balkowiec et al., 2000; Woo et al., 2005) or GABAergic (Brünig et al., 2001; Henneberger et al., 2005) synapses. In the highly plastic immature nervous system, these presynaptic and postsynaptic changes are expected to affect the number and function of synapses as well as the shape of respective dendrites; however, the source of BDNF, the signaling pathways involved, and the extent of structural changes exerted by the activation of NTRs are not yet fully elucidated. Even the general principles governing preferential formation or stabilization of excitatory as opposed to inhibitory synaptic input are far from being clear.

Most previous observations were based on experiments with 
chronic BDNF deficiency, on experiments with application of exogenous BDNF, or experiments with BDNF overexpression. The latter approach has the advantage that BDNF may be released from a natural source, but the cellular environment of the transfected neurons is undefined with respect to neurotrophin expression and release. This makes it difficult to assign an effect to a particular signaling route. We therefore used hippocampal neuronal cultures from $b d n f-/-$ mice to establish an unequivocal postsynaptic source of BDNF by short-time expression of BDNF::enhanced green fluorescent protein (EGFP) in a very small number of neurons.

The specific aims of the present experiments were as follows: (1) to compare the ability of BDNF-expressing and nonexpressing neurons to attract BDNF-negative axon terminals, (2) to determine the differential action of postsynaptic BDNF on glutamatergic as opposed to GABAergic synaptic inputs, and (3) to find out to what extent BDNF-dependent alterations of dendrite morphology were caused by changes in synapse number or activity; finally (4), we sought to clarify whether p $75^{\mathrm{NTR}}$-dependent processes can contribute to the synaptic or dendritic plasticity of hippocampal neurons at the early stages of circuit development.

\section{Materials and Methods}

Animals, preparation, and transfection. Our $b d n f$ knock-out mice colony was raised from two pairs of $b d n f+/-$ mice in a C57BL/6 background (Ernfors et al., 1994) purchased from The Jackson Laboratory (Bar Harbor, ME). These mice have a low rate of pregnancy and few pups per litter. Therefore, to obtain a sufficient number of $b d n f-/-$ embryos from each pregnant mother, we generated a new line of mice by crossing $B L / 6 / b d n f+/$ - with CD1 mice, following the procedure of Pozzo-Miller et al. (1999). To prepare hippocampal cultures from embryonic day 18 (E18) mice, the embryos were rapidly genotyped (Henneberger et al., 2000), and the hippocampi from all embryos were kept in ice-cold PBS. Once the genotype was confirmed, all subsequent steps followed the protocol of Goslin et al. (1998). Briefly, cells were dissociated after digestion with $0.5 \%$ trypsin (Worthington, Lakewood, NJ) and DNase I (Sigma, St. Louis, MO), plated on glass coverslips coated with poly-Lornithine (Sigma), and cultured in Neurobasal A supplemented with B-27 (both from Invitrogen, Crewe, Cheshire, UK), $\beta$-mercaptoethanol $(12.5 \mu \mathrm{M})$, L-glutamine $(250 \mu \mathrm{M})$, penicillin $(5 \mu \mathrm{g} / \mu \mathrm{l})$, streptomycin $(12.5 \mu \mathrm{g} / \mu \mathrm{l})$, and $1 \%$ FCS. Twenty-four hours after plating, the growth medium was half-exchanged with fresh medium. Unless stated otherwise, the initial cell density was 75,000 per square centimeter. Under the conditions used, our cultures were nearly devoid of glia.

On $7 \mathrm{~d}$ in vitro (DIV7), the neurons were transfected with Effectene Transfection reagent (Qiagen, Hilden, Germany), following the manufacturer's instructions. To establish a local cellular source of BDNF in a BDNF-free cellular environment, a small fraction $(\sim 0.02 \%)$ of cultured hippocampal neurons from $b d n f-/-$ mice was transfected with an EGFP- or BDNF::EGFP-expressing N1 vector (Clontech, Cambridge, UK), as described by Haubensak et al. (1998). The expression times were kept short (16 h, unless mentioned otherwise). During this time, the cultures were maintained in either control medium or medium containing BDNF $(100 \mathrm{ng} / \mathrm{ml})$. In part of the experiments, the medium was supplemented with a blocking antibody against $\mathrm{p} 75^{\mathrm{NTR}}$ (p75-Ab, 1:500; Advanced Targeting Systems, San Diego, CA), TrkB (TrkB-Ab, 1:500; BD Biosciences, Heidelberg, Germany), the tyrosine kinase inhibitor K252a (200 nM; Sigma), or a mixture of glutamate receptor (GluR) antagonists. Unless indicated otherwise, the GluR blocking mixture consisted of 6,7dinitroquinoxaline-2,3(1H,4H)-dione (DNQX; $20 \mu \mathrm{M})$, MK801 (1 $\mu \mathrm{M})$, and $(R S)$ - $\alpha$-methyl-4-carboxyphenylglycine $(200 \mu \mathrm{M})$ (all from Tocris, Bristol, UK).

After the indicated expression and incubation time, the cultures were fixed for immunostaining (4\% paraformaldehyde/4\% sucrose in PBS), prepared for real-time PCR, or used for electrophysiological recording of synaptic activity. Cultures displaying uneven cell distributions or deviating from the expected neuron density on DIV6 $(60,000$ per square cen- timeter) were not included. Only nonoverlapping EGFP + neurons (distance from next labeled soma $>2000 \mu \mathrm{m}$ ) with apparently healthy dendrites (not beaded and no dead-end thick branches) were selected for further analysis. Typically, 10-20 neurons were studied in one coverslip (culture area, $0.785 \mathrm{~cm}^{2}$ ).

Because of the young age at dissociation and the short time in vitro, neurons did not reach full maturity, but dendrites could be distinguished from axons. On DIV6, neurons had only one axon, and microtubuleassociated protein 2 (MAP2) immunoreactivity covered $\sim 90 \%$ of the dendritic length (see Fig. $5 a$ ). The dendrites of $b d n f-/-$ hippocampal neurons were functional, because they were able to form axodendritic synaptic contacts (see Fig. 1b) and to generate postsynaptic currents (see Fig. 4a). Morphological criteria could not be used for identification of a particular phenotype because experimental manipulations themselves exerted an effect on neuron shape (see Fig. $5 a$ ).

Immunocytochemistry, image acquisition, and morphometric analysis of labeled hippocampal neurons. The following primary antibodies were used: mouse anti-MAP2 (1:400; Sigma), rabbit anti-GFP (1:100; Invitrogen, Eugene, OR), chicken anti-GFP (1:500; Chemicon, Temecula, CA), mouse anti-GFP (1:100; BD Biosciences, San Jose, CA), mouse antisynaptophysin I [(Syp) 1:50; Progene, Heidelberg, Germany], or antisynapsin I [(Syn) Chemicon, Hampshire, UK], guinea pig anti-vesicular glutamate transporter 1 and 2 (VGluT1/2; Chemicon), and rabbit antivesicular inhibitory amino acid transporter [(VIAAT) 1:500; Synaptic Systems, Göttingen, Germany]. To verify that the labeling was caused specifically by the primary antibodies, it was either omitted or replaced by similarly diluted normal serum from the same species. Appropriate secondary antibodies, fluorochromes, and filters were selected for double, triple, or quadruple immunofluorescence analysis.

Our methods for the evaluation of dendritic morphology and presynaptic terminal identification in dissociated cell cultures have been described previously (Henneberger et al., 2005; Salama-Cohen et al., 2005, 2006). Briefly, labeled neurons were visualized by standard epifluorescence under a Plan-Neofluar $63 \times$ oil objective with a numerical aperture of 1.3 (Zeiss, Oberkochen, Germany). Images were acquired with a 12-bit cooled CCD camera $[(1317 \times 1035$ pixels $)$ CH250; Photometrics, Tucson, AZ)] with software PMIS 4.1.4 software (Remington, Munich, Germany). Terminal counts and analysis of dendrite morphology were performed manually or with the help of in-house software written by C. Henneberger. With the magnification used, the pixel area was $0.104 \times$ $0.104 \mu \mathrm{m}$. A circular region of interest (ROI) with a diameter of $100 \mu \mathrm{m}$ was projected onto the EGFP-labeled neuron, its center roughly coinciding with the center of the soma. The dendrite length was expressed as the number of dendritic trees that exceeded the limit of the ROI (number of dendrites, $>50 \mu \mathrm{m}$ ). The branching pattern was characterized by the number of primary dendrites, i.e., the number of dendrites associated with the soma, and the number of dendritic branch points per ROI. A branch point was defined as the point of origin of at least two daughter branches with a length $>7 \mu \mathrm{m}$. Synaptic terminals in contact with an EGFP-labeled neuron were identified by single immunofluorescence, when an antibody against Syp, VGluT, or VIAAT was used, or by double fluorescence (typically VGluT, VIAAT, and Syp). To avoid false positives or negatives in the synaptic terminal counts, we also used a size criterion. Assuming a round shape of the contact, a fluorescent spot was required to have suprathreshold intensity values over an area with a diameter between 0.5 and $2 \mu \mathrm{m}$, the threshold intensity being equal to the average intensity of the ROI plus three times its SD. Contamination of VGluT or VIAAT immunofluorescence with the fluorescence produced by Syp or Syn staining was assessed by using identical ROIs centered on Syp or Syn + puncta devoid of apparent VGluT or VIAAT staining. The respective ratios were $<0.05$ in all ROIs that were evaluated.

For evaluation of synaptic terminals in the hippocampus of wild-type and BDNF-deficient mice, frontally cut $500 \mu \mathrm{m}$ brain slices were fixed in $4 \%(\mathrm{w} / \mathrm{v})$ paraformaldehyde and $4 \%(\mathrm{w} / \mathrm{v})$ sucrose in PBS, stored for $6 \mathrm{~h}$ at $4^{\circ} \mathrm{C}$, washed three times in PBS, cryoprotected overnight at $4^{\circ} \mathrm{C}$ in PBS containing $8 \%$ sucrose, and embedded in Tissue-Tek OCT compound (Sakura Finetek, AT Zoeterwoude, The Netherlands). Sections of $20 \mu \mathrm{m}$ were cut with a cryomicrotome (Jung CM 1800; Leica), and 8-10 consecutive sections per genotype and animal were prepared from a total of 
six animals and processed for immunocytochemistry. After three washes in PBS and one wash in PBS containing $0.1 \%(w / v)$ gelatin, the sections were incubated for $12 \mathrm{~h}$ at $4^{\circ} \mathrm{C}$ in the primary antibodies diluted in PBS-gelatin supplemented with $0.12 \%(w / v)$ Triton X-100. After extensive washes in PBS-gelatin, the secondary antibodies were applied for $1 \mathrm{~h}$ at room temperature in PBS-gelatin. To verify that the labeling was specific, the primary antibodies were replaced by similarly diluted normal serum from the respective species.

Immunoreactivity was visualized with a Leica DM IRBE confocal microscope (Leica Camera AG, Solms, Germany) equipped with an argonkrypton laser. Cyanine 3 and fluorescein fluorochromes were visualized with excitation wavelengths of 568 and $488 \mathrm{~nm}$, respectively. Doublelabeled sections were scanned by the simultaneous application of both excitation wavelengths. Controls included dual-channel recordings of single-labeled sections, as well as dual-channel recordings of doublelabeled single antigens to detect bleed-through phenomena and possible shifts between the detected signals. Fluorescent images were always derived from the same region, as described in the legend to supplemental Figure 1 (available at www.jneurosci.org as supplemental material). The images were background subtracted, scaled according to maximum and minimum fluorescence intensities, and subsequently thresholded. The number of terminals per ROI $(100 \times 100 \mu \mathrm{m})$ was determined by an automated routine of the NIH software image. Immunolabeled puncta were classed as terminals if (1) the fluorescence intensity exceeded onethird above the minimal fluorescent level (2) and the cross-section area exceeded $0.2 \mu \mathrm{m}^{2}$. The data are presented as mean \pm SEM and tested for significance by one-way ANOVA with the software Origin 7.5 (Microcal, Northampton, MA).

Quantitative real-time PCR. Total RNA was extracted from DIV6 cultures with Trizol reagent (Invitrogen, Karlsruhe, Germany). cDNA synthesis was performed by using $2 \mu \mathrm{g}$ of RNA with Superscript II reverse transcriptase (Invitrogen). Quantitative PCR was performed with the ABI Prism 7000 Sequence Detector (Applied Biosystems, Weiterstadt, Germany), following the supplier's instructions, in a $20 \mu$ l volume reaction with the SYBR Green PCR Master Mix for Syp and VIAAT and the Taqman Master Mix for the control housekeeping gene, gapdh. All reactions were performed in triplicates, and the experiments were repeated at least three times. The TaqMan probes (labeled with 6-carboxy fluorescein) and primers for gapdh were Assay-on-Demand gene expression products (Applied Biosystems). The standard curves for Syp and gapdh were generated with serial dilutions of cDNAs from DIV6 hippocampal cultures. Target gene expression was normalized for gapdh expression. The $5^{\prime}-3^{\prime}$ primer sequence for Syp is given as follows: TGCTACGTGCGGCAGCTA (forward) and TCTCCGTCTTGTTGGCACACT (reverse). The $5^{\prime}-3^{\prime}$ primer sequence for VIAAT is AATTTTGGCTCCTGTCAACGA (forward) and CACCCTCAAGGTCAAGTTTCCA (reverse).

Electrophysiology. Patch-clamp experiments were performed on EGFP-labeled or nonlabeled $b d n f-/-$ neurons (control) and on BDNF::EGFP-transfected neurons. The time after transfection was $70-80 \mathrm{~h}$. The culture medium was exchanged by an extracellular solution containing (in $\mathrm{mm}$ ) $150 \mathrm{NaCl}, 3 \mathrm{KCl}, 1 \mathrm{MgCl}_{2}, 2 \mathrm{CaCl}_{2}$, and 35 glucose; $\mathrm{pH}$ was adjusted to 7.4 with $\mathrm{NaOH}$. Cultures were allowed to equilibrate in a recording chamber mounted on the stage of an Axioskop 2 FS plus (Zeiss) for $30 \mathrm{~min}$ before recording. Transfected cells were visualized with epifluorescence at an excitation wavelength of $480 \mathrm{~nm}$ (Till Photonics, Munich, Germany). Miniature EPSCs (mEPSCs) and mIPSCs were isolated pharmacologically by blocking NMDA receptors with DL-aminophosphonovaleric acid (100 $\mu \mathrm{M}$; Sigma), blocking glycine receptors with strychnine ( $0.4 \mu \mathrm{m}$; Sigma), and blocking action potential generation with tetrodotoxin ( $1 \mu \mathrm{M}$; Alomone Labs, Jerusalem, Israel) and were identified by the respective receptor blockers DNQX $(20 \mu \mathrm{M})$ and gabazine $(3 \mu \mathrm{M})$ (both from Tocris). Synaptic activity was recorded with an EPC-8 amplifier (List Electronics, Darmstadt, Germany), in the whole-cell configuration, at a holding voltage of $-60 \mathrm{mV}$ at room temperature during a period of $0.5-2 \mathrm{~h}$ after removal of the culture from the incubator. The pipette solution contained the following (in $\mathrm{mm}$ ): 145 $\mathrm{CsCl}, 10$ EGTA, 20 HEPES, $5 \mathrm{NaCl}, 1 \mathrm{CaCl}_{2}$, and $2 \mathrm{MgCl}_{2}$, pH 7.2. The pipette-to-bath DC resistance of patch electrodes ranged from 4 to $6 \mathrm{M} \Omega$ (tip diameter, $1-2 \mu \mathrm{m})$. Series resistance $\left(R_{\mathrm{s}}\right)$ was monitored regularly and compensated $>70 \%$. Recordings were rejected if $R_{\mathrm{s}}$ exceeded $25 \mathrm{M} \Omega$ or the change of $R_{\mathrm{s}}$ exceeded $30 \%$. Signals were sampled at a rate of 10 $\mathrm{kHz}$ with a 16-bit AD converter and WinTida 4.11 software (HEKA Elektronik, Lambrecht, Germany). Postsynaptic currents were low-pass filtered at $3 \mathrm{kHz}$ and analyzed with in-house software written by $\mathrm{C}$. Henneberger. The time of occurrence, the maximal peak amplitude, and the $20-80 \%$ rise time were computed. The decay of mPSCs could be well fitted by a single exponential function.

Culture materials and statistical analysis. Statistical analysis was performed with Origin 7.5 software (Microcal). All experiments were designed to compare neurons entirely devoid of BDNF with neurons exposed to BDNF in any of three ways: addition of exogenous BDNF, transfection of BDNF into a small number of neurons for 16 or $72 \mathrm{~h}$, or natural (undefined) expression in cultures from wild-type mice. Thus, the "control" cultures were always the untreated cultures from $b d n f-/-$ mice. The $b d n f-/-$ cultures used for comparison were obtained from one or several embryos of the same mother and prepared and transfected on the same day. Naturally, the $b d n f+/+$ cultures had to be prepared separately, but care was taken to produce cultures matching the $b d n f-/-$ controls as closely as possible. Typically, two coverslips were prepared per condition in one experiment, and 5-15 neurons were evaluated on each coverslip. The total number of coverslips and neurons per condition is given in the figures above each bar. For statistical analysis, the neurons were pooled from different coverslips. This is justified by the absence of significant differences between the coverslip means, as tested by ANOVA (see Fig. $1 c, d$ ). In this case, we first tested whether the coverslip averages were distributed normally at the significance level of 0.05 (Shapiro-Wilk test). Only $10-15 \%$ of the coverslips did not pass this test. If not stated otherwise, the pooled data are presented as mean \pm SEM. To test for significant differences between two conditions, we applied the Student's $t$ test. For comparison of three or more conditions, we applied three tests: (1) Nevene's test for equal variance, (2) ANOVA, and (3) post hoc Bonferroni's test for significant differences between two groups. The significance levels shown in the figures apply to the results of the Student's $t$ test or the Bonferroni's test. The horizontal bars indicate which conditions were compared, and asterisks indicate the significance levels: ${ }^{*} p<0.05$, ${ }^{* *} p<0.01$, and ${ }^{* * *} p<0.001$.

\section{Results \\ BDNF expression in the postsynaptic neuron facilitates synapse formation via TrkB but not $\mathrm{p} 75^{\mathrm{NTR}}$}

Previous studies from other laboratories have reported an increase in the expression of synaptic markers or the number of synaptic terminals under the influence of exogenous BDNF added to wild-type hippocampal neurons (Vicario-Abejon et al., 2002; Yang et al., 2004). For comparison with these studies, we examined the results of chronic BDNF deficiency or the addition of exogenous BDNF to cultures from $b d n f-/-$ mice. Quantitative PCR experiments revealed that hippocampal cultures from $b d n f-/-$ mice displayed a significantly lower level of Syp mRNA than cultures from wild-type mice (Fig. 1a); however, the addition of exogenous BDNF (100 ng/ml; $16 \mathrm{~h}$ ) raised Syp expression to a level even exceeding that of wild-type cultures.

By applying an antibody against Syp, we identified synaptic terminals in contact with EGFP- or BDNF::EGFP-labeled neurons in wild-type or $b d n f-/-$ cultures (Fig. 1b). To obtain reliable estimates of terminal numbers per ROI and EGFP-labeled neurons, the results were pooled from a relatively large number of coverslips per condition. The absence of significant differences between the coverslip means were tested by ANOVA (Fig. 1c,d). The pooled data were distributed almost normally (Fig. 1e,f). Comparison of the means by ANOVA and post hoc Bonferroni's test revealed significant differences between the control neurons, i.e., untreated EGFP-labeled $b d n f-/-$ neurons, and test neurons, i.e., EGFP-labeled $b d n f-/-$ neurons that were treated with exogenous BDNF, transfected with BDNF::EGFP, or derived from 
a

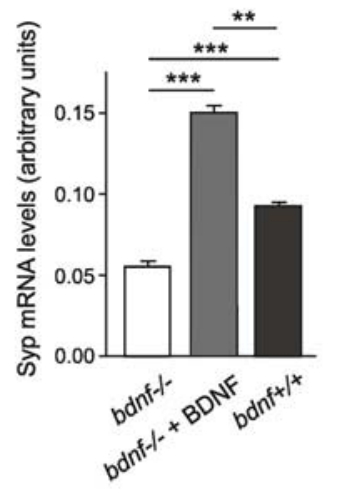

b
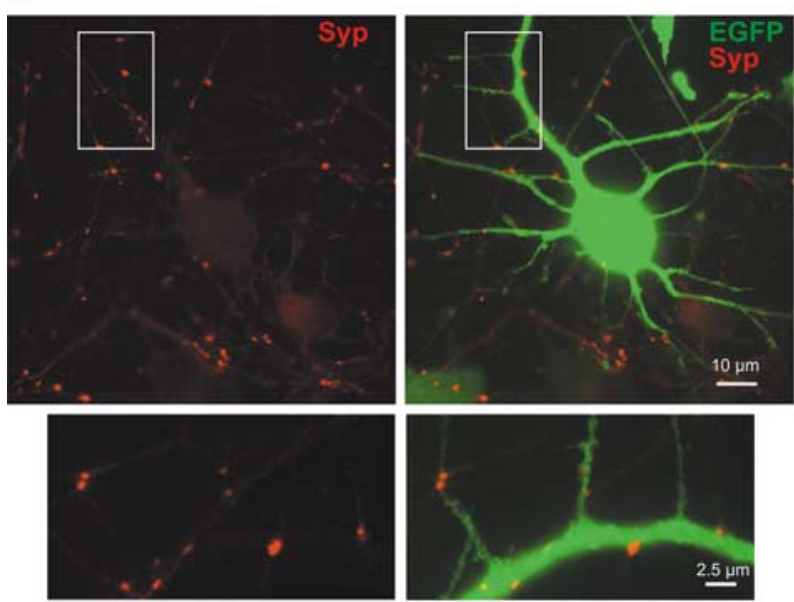

C

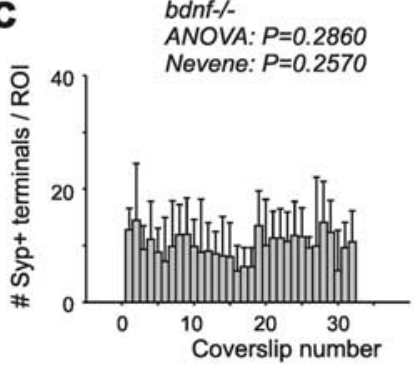

$\mathbf{e}$

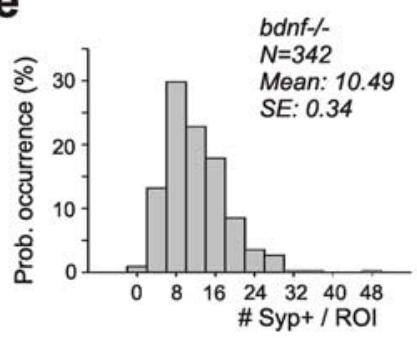

g

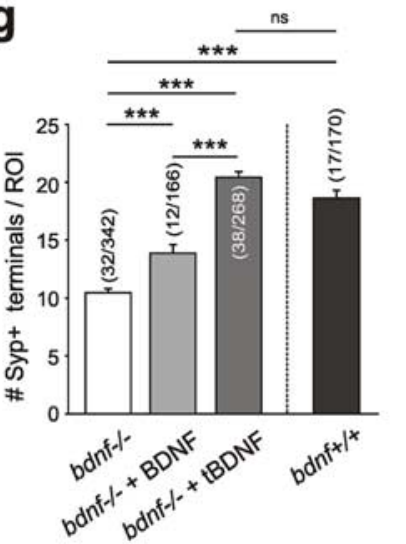

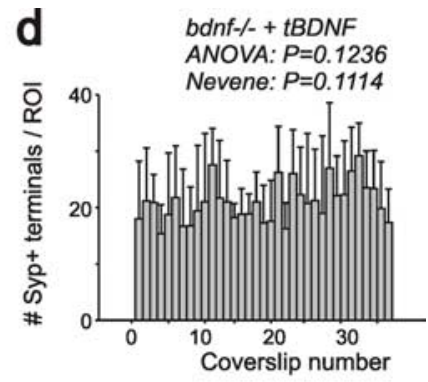

f

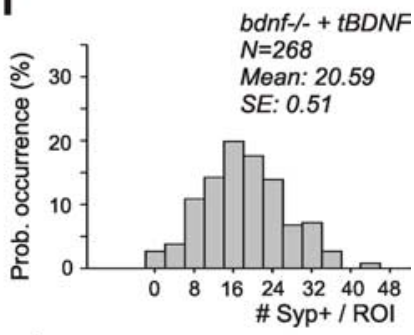

h

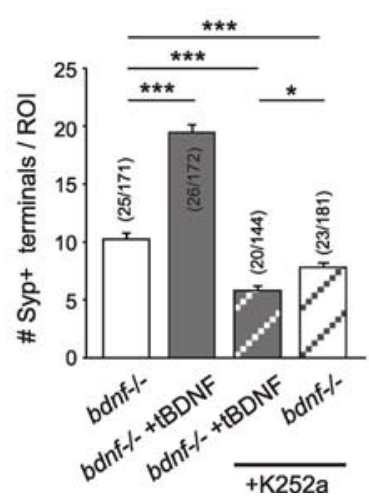

Figure 1. TrkB-dependent upregulation of synaptic terminal numbers by postsynaptic BDNF. $\boldsymbol{a}$, Real-time PCR analysis of BDNF-dependent Syp gene expression. E18 hippocampal neurons were dissociated from bdnf $+/+$ or $b d n f-/-$ mice and cultured at an initial cell density of 75,000 cells per square centimeter. After $5 \mathrm{~d}$ in culture, cells were exposed for $16 \mathrm{~h}$ to either control medium or medium supplemented with BDNF $(100 \mathrm{ng} / \mathrm{ml})$. Cells were then harvested for RNA extraction, and the transcript levels of Syp were determined by real-time PCR. The chronic absence of BDNF reduced the levels of Syp mRNA and the addition of exogenous BDNF increased it. $\boldsymbol{b}$, Immunofluorescence images of an EGFP-expressing neuron from E18 bdnf - / - cultures after immunostaining with an antibody against Syp. $\boldsymbol{c}, \boldsymbol{d}$, Counts of Syp + terminals in contact with an EGFP-labeled neuron. ROI size: $100 \times 100 \mu \mathrm{m}$. Typically 5-15 neurons were evaluated on each coverslip. Error bars indicate mean \pm SD in each coverslip. Note the absence of significant differences between coverslips in tests with ANOVA. $\boldsymbol{e}, \boldsymbol{f}$, Histograms of the data pooled from cand $\boldsymbol{d}$. The mean \pm SE of these data sets are shown in the first and third bar in $\boldsymbol{g}$. For further details of statistical evaluation and meaning of symbols, see Materials and Methods. $\boldsymbol{g}$, $\boldsymbol{h}$, Quantification of results obtained from sister cultures from BDNF-deficient hippocampi. The data for wild-type neurons are from a different set of experiments, but the cultures were prepared in the same way as the cultures from knock-out mice. Note the increase in the number of Syp + terminals after addition of exogenous BDNF ( $50-100 \mathrm{~nm}$ for $16 \mathrm{~h})$, BDNF transfection (expression time, $16 \mathrm{~h}$ ), and absence of BDNF effects in neurons treated during the expression time with K252a (200 nM). ${ }^{*} p<0.05 ;{ }^{* * *} p<0.001$. Prob. occurrence, Probability of occurrence; ns, not significant.

wild-type animals (Fig. 1g). Interestingly, the innervation level reached after BDNF transfection was higher than that obtained with exogenous BDNF $(100 \mathrm{ng} / \mathrm{ml})$ in the medium. Under the conditions chosen, BDNF transfection restored nearly exactly the terminal numbers observed in wild-type preparations. These results emphasize the widely accepted hypothesis that local availability of neurotrophins rather than even distribution of large amounts of ambient BDNF must account for the strong synaptotropic effect of this neurotrophin in the developing CNS (Vicario-Abejon et al., 2002; Yang et al., 2004).

It is noteworthy that immunostaining of hippocampal sections at postnatal day 3 showed no differences in the total terminal numbers (VGluT + and VIAAT + ) between wild-type and $b d n f$ knock-out mice (supplemental Fig. 1, available at www. jneurosci.org as supplemental material), suggesting that other factors could compensate for the absence of BDNF in an embryo. This discrepancy is one more piece of evidence showing that global comparison of terminal numbers in wild-type and knockout animals is not necessarily the best way to unravel the capacity of this neurotrophin to regulate synaptogenesis. It should be pointed out, however, that the differences found in Syp + terminal numbers between BDNF-deficient and BDNFtransfected neurons were not associated with differences in neuron density, because the counts were performed in density-matched cultures.

To determine the type of neurotrophin receptors in this form of synaptic plasticity, we applied the tyrosine kinase inhibitor K252a (Fig. 1h). K252a is not a specific blocker of TrkB. Because it could have effects that were unrelated to BDNF, we also tested its action on $b d n f-/-$ neurons. It was found that the transfected BDNF (tBDNF)-induced increase in Syp + terminal number was prevented by the tyrosine kinase inhibitor. An antibody against TrkB was tested in a small number of neurons and had similar effects (supplemental Fig. $2 c$, available at www.jneurosci. 

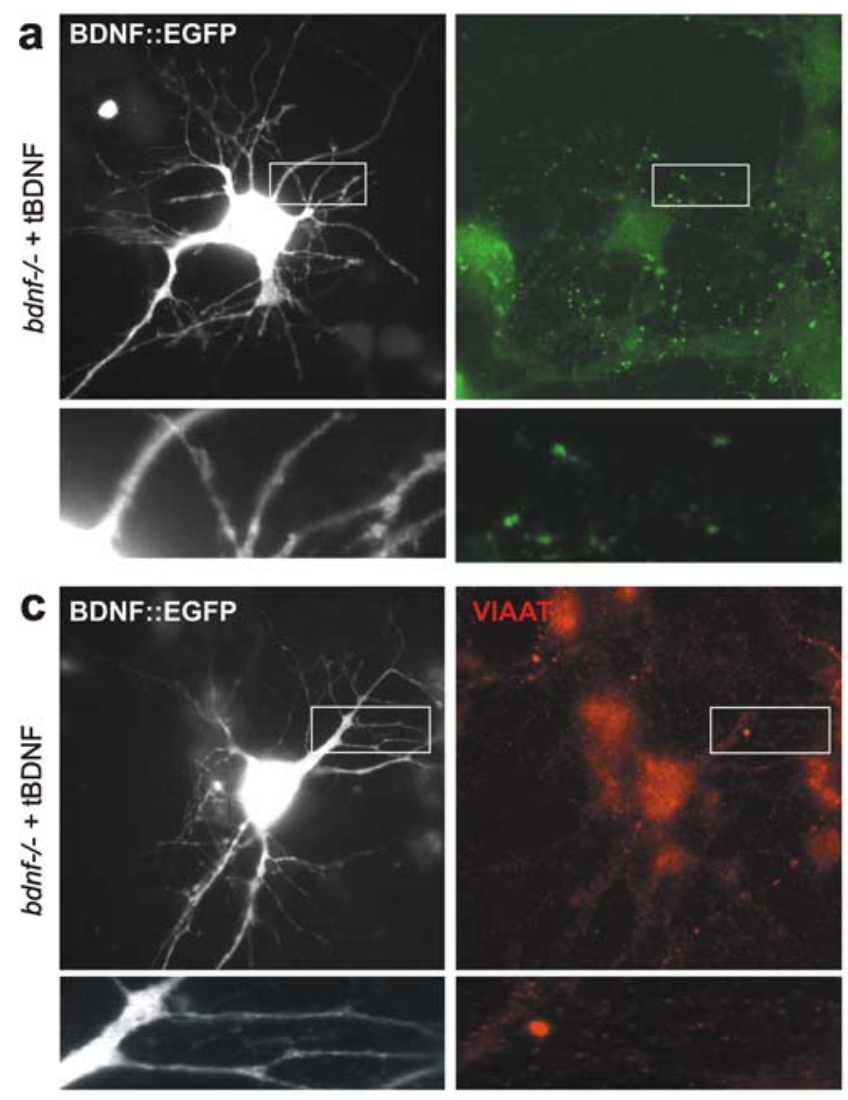
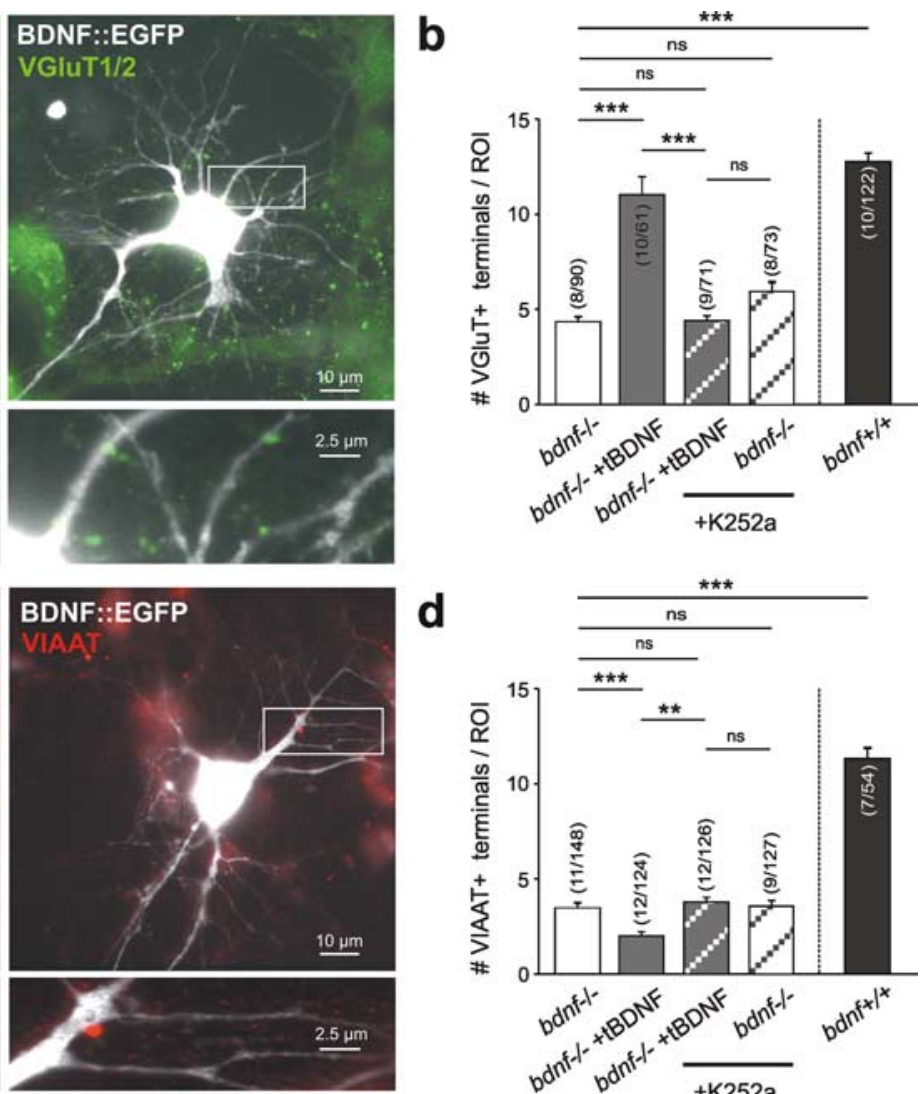

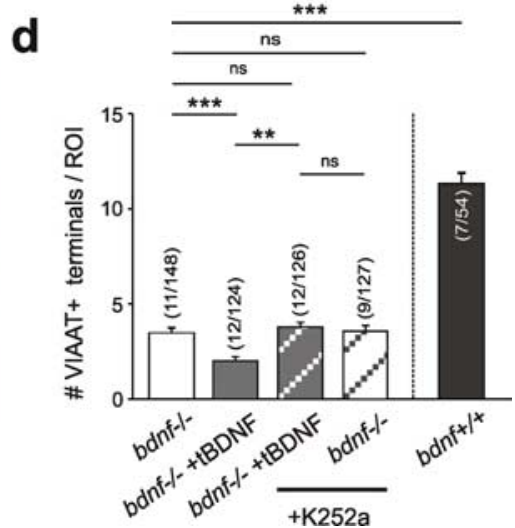

Figure 2. Enhanced glutamatergic, but not GABAergic synaptic terminal numbers in solitary BDNF-expressing neurons in a BDNF-deficient environment. $\boldsymbol{a}$, $\boldsymbol{c}$, Immunofluorescence images of transfected neurons after $16 \mathrm{~h}$ expression of BDNF::EGFP. Pseudocolors show EGFP in white, VGluT1/2 in green, and VIAAT in red. That the synaptic terminals were also stained with an antibody against Syp and visualized with Alexa 350 is not shown. Boxed areas are shown enlarged below the corresponding image. $\boldsymbol{b}, \boldsymbol{d}$, Quantification of results. Both the tBDNF-induced upregulation of VGluT + synaptic terminal numbers and the decrease in VIAAT + terminal numbers are prevented by incubation in K252a (200 nm). Cultures prepared from wild-type mice displayed an appreciably higher number of VGluT + and VIAAT + terminals per neuron and ROI; however, the respective cultures had to be prepared separately. ${ }^{* *} p<0.01 ;{ }^{* * *} p<0.001$. ns, Not significant.

org as supplemental material), whereas an antibody against p75 ${ }^{\mathrm{NTR}}$ (Huber and Chao, 1995) failed to change the number of Syp + terminals (supplemental Fig. 2e, available at www.jneurosci.org as supplemental material); however, in contrast to the blocking effect of p75-Ab on synaptic responses to exogenous NGF (Salama-Cohen et al., 2006), the synaptogenic action of tBDNF remained unaltered.

Postsynaptic BDNF is an attractant for glutamatergic synaptic terminals, but it suppresses the formation of GABAergic synapses

The effects of tBDNF may be limited to a subpopulation of synaptic terminals. We therefore asked to what extent glutamatergic as opposed to GABAergic synaptic terminals benefited from the presence of tBDNF in the postsynaptic neuron. Double immunostaining was performed with antibodies against VGluT1/2 and Syp or VIAAT and Syp. Figure 2, $a$ and $c$, shows specimens of VGluT + and VIAAT + terminals in contact with BDNF::EGFP-expressing neurons in a BDNF-deficient cellular environment. Here and in the following text we shall refer to VGluT + (and Syp + ) and VIAAT + (and Syp +) axodendritic or axosomatic terminals as glutamatergic and GABAergic synaptic terminals, respectively. Assuming that these hippocampal cultures lack glycinergic synapses (Meier and Grantyn, 2004), VIAAT would solely identify GABAergic synaptic terminals. Quantification of the immunostained preparations revealed that the presence of BDNF had strikingly different consequences for glutamatergic, as opposed to GABAergic, synaptic terminals (Fig. $2 b, d$ ).
Compared with the BDNF-deficient controls, neurons expressing tBDNF displayed an increase in the number of VGluT + terminals, reaching $11.03 \pm 0.94$ glutamatergic terminals per ROI and neuron. This is close to the level observed in densitymatched wild-type cultures. The upregulation was completely prevented by the tyrosine kinase inhibitor K252a (Fig. 2b) or the antibody against TrkB (data not shown). In contrast with this tBDNF-induced facilitation of glutamatergic synaptogenesis, the number of GABAergic synaptic terminals failed to increase. On the contrary, the counts revealed a significantly lower number of VIAAT + synaptic terminals in neurons expressing tBDNF (Fig. $2 c$ ). Again, the tBDNF-induced changes were prevented by TrkB blockade. However, despite this depressant effect of postsynaptic tBDNF on the number of VIAAT + terminals, the overall density of inhibitory synaptic terminals was clearly lower in $b d n f-/-$ cultures as opposed to $b d n f+/+$ cultures.

A previous study from our laboratory (Henneberger et al., 2005) has shown that added BDNF increases the level of GAD65 in synaptic terminals, but it decreases the level of VIAAT. To determine whether the latter effect is also detectable at the level of mRNA, we performed quantitative reverse transcriptase-PCR experiments. It was found that the addition of exogenous BDNF to $b d n f-1-$ cultures resulted in a marked decrease in VIAAT mRNA (supplemental Fig. $2 a$, available at www.jneurosci.org as supplemental material), although the VIAAT mRNA levels were higher in bdnf-/- mice.

Thus, it became clear that neurons able to express and release 
a
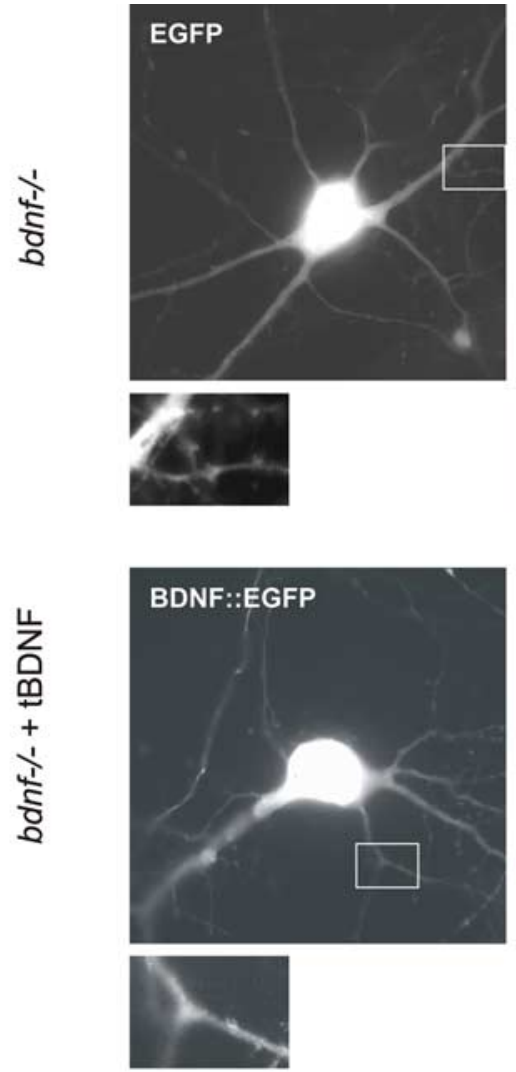
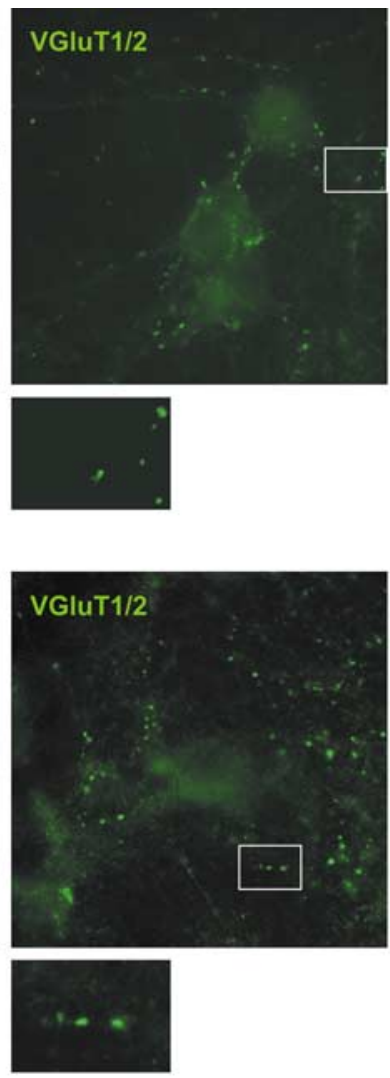
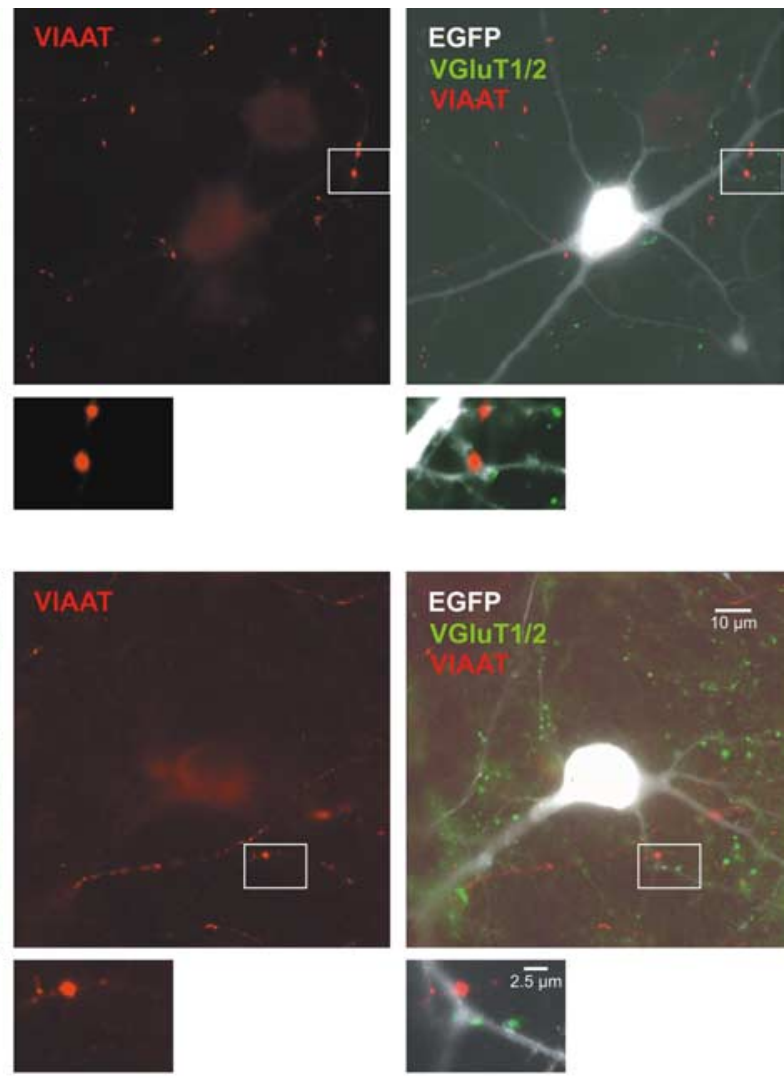

b

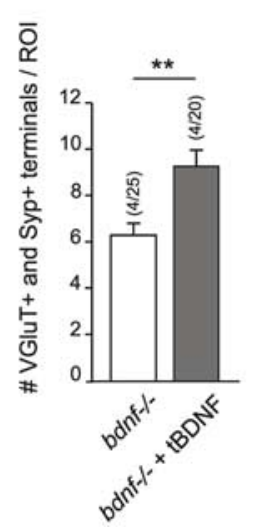

$\mathbf{c}_{\bar{\alpha}}$

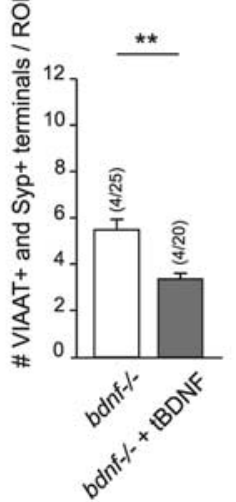

d

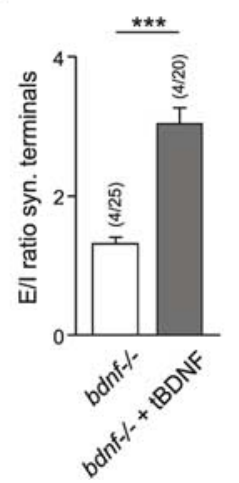

e

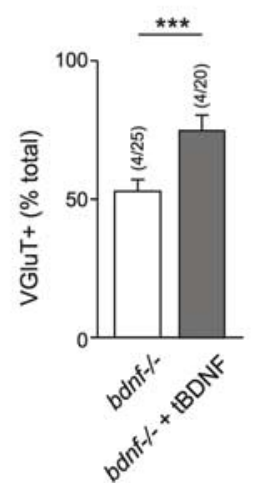

Figure 3. Enhanced ratio of E/I synaptic terminal numbers in BDNF-expressing neurons. $\boldsymbol{a}$, Immunofluorescence images of transfected DIV6 neurons after $72 \mathrm{~h}$ expression of EGFP (top panel) or BDNF::EGFP (bottom panel). Pseudocolors show EGFP in white, VGluT in green, and VIAAT in red. Syp immunofluorescence is not shown. $\boldsymbol{b}-\boldsymbol{e}, 0$ uantification of results. ${ }^{* *} p<0.01 ;{ }^{* * *} p<0.001$.

high amounts of BDNF are prone to an increased ratio of excitatory to inhibitory (E/I) synaptic input. Consequently, BDNFexpressing neurons should make a larger contribution to the developing network activity. On the other hand, it is well known that, in the long run, neurons tend to converge to an intermediate level of network activity, first of all by adjusting the number of postsynaptic receptors (Wierenga et al., 2005). One might therefore expect that the obvious imbalance between $\mathrm{E}$ and I terminal numbers is only transient. To address this point we increased the expression times to $72 \mathrm{~h}$ and determined the E/I ratio of individual neurons by performing quadruple immunofluorescence staining against VGluT and VIAAT, in addition to Syp (data not shown) and EGFP (Fig. $3 a$ ). The graphs in Figure $3 b-e$ show that, despite the prolonged expression times, tBDNF neurons at- tracted a larger number of glutamatergic and a lower number of GABAergic synaptic contacts. Accordingly, the average E/I ratio of synaptic input (Fig. 3c) and the fraction of glutamatergic terminals (compare Fig. $3 e$ with Fig. $4 e$ ) were higher in BDNFexpressing neurons.

To determine whether these alterations were matched by the electrophysiological characteristics of spontaneous transmitter release, miniature synaptic activity was recorded on DIV8, 72-80 $\mathrm{h}$ after transfection. At this age, all neurons generated a mixture of rapidly and slowly decaying mPSCs (Fig. 4a), but the frequency of mPSCs varied to a great extent from neuron to neuron (0.023$0.873 \mathrm{~Hz}$ ). The distribution of the time constants of mPSC decay $\left(\tau_{\mathrm{D}}\right)$ was plotted as a double Gaussian function for each cell. The two peaks displayed a differential sensitivity to the GluR blocker 
a

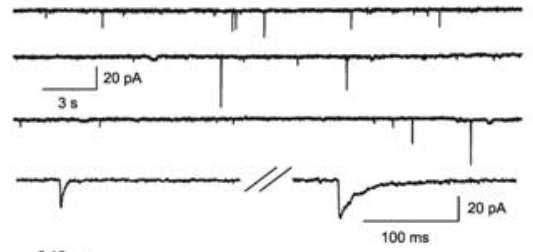

b
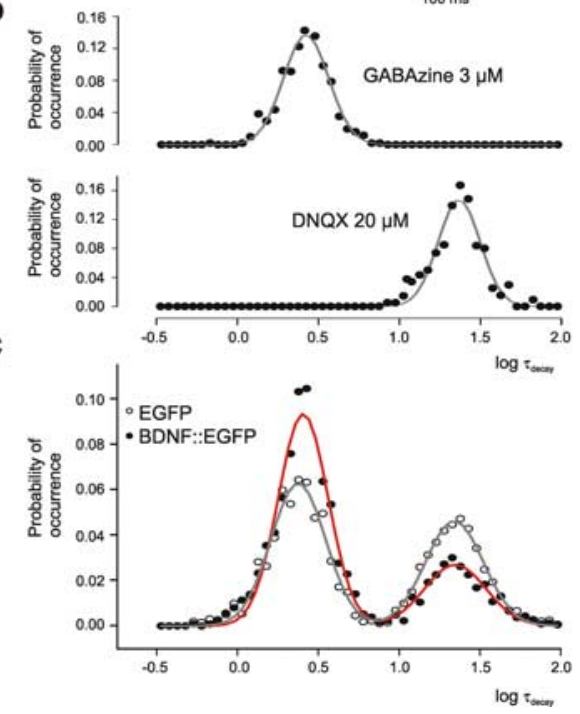

d
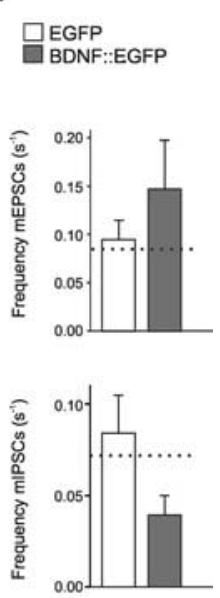

e

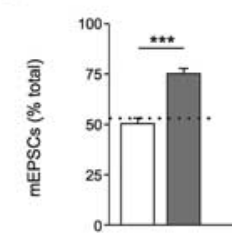

Figure 4. The presence of tBDNF in the postsynaptic neuron increases the fraction of glutamatergic mIPSCs. mPSCs were recorded from cultured hippocampal neurons in bdnf-/- hippocampal cultures at DIV8. The controls lacked BDNF and either were not transfected ( $n=11$ ) or they expressed EGFP only $(n=18)$. The test neurons $(n=14)$ expressed BDNF::EGFP. $\boldsymbol{a}$, Sample traces of mPSC recording. Two populations of mPSCs could clearly be distinguished by their decay kinetics. $\boldsymbol{b}$, Single Gaussian distributions of $\log \tau_{\text {decay }}$ in the presence of the GABA receptor blocker gabazine ( $3 \mu \mathrm{m}$ ) (top graph; $n=4)$ or the GluR blocker DNQX (20 $\mu \mathrm{m}$ ) (bottom graph; $n=4)$. c, Double Gaussian functions could be well fitted to the experimental data acquired in the absence of DNQX and gabazine. The point of interception of the two Gaussian functions corresponds to a $\tau_{\text {decay }}$ of $7.4 \mathrm{~ms}$. This threshold was used to discriminate between

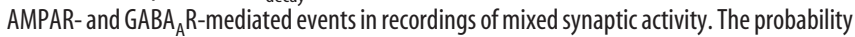
of erroneously attributing a postsynaptic event to either $G_{A B A_{A} R}$ or AMPAR activation was calculated to be $0.31 \%$. $\boldsymbol{d}$, Effect of transfected BDNF on the frequency of mEPSCs or mIPSCs. The control values obtained from nontransfected $b d n f-/-$ neurons are indicated by the dashed line. e, BDNF-expressing neurons display an increased fraction of glutamatergic mPSCs (frequency of $\mathrm{mEPSC} / \mathrm{mPS}$ ( frequency).

DNQX and the GABA $\mathrm{A}$ blocker gabazine (Fig. $4 b$ ) and therefore were assumed to represent glutamatergic mEPSCs and GABAergic mIPSCs, respectively. It was found that the presence of tBDNF increased the probability of occurrence of events with a short time constant of mPSC decay (Fig. 4c). Quantification of the results revealed a tendency of the mEPSC frequency to increase and of the MIPSC frequency to decrease under the influence of BDNF expression (Fig. $4 d$ ). The fraction of mEPSCs was significantly higher in BDNF-expressing neurons (Fig. 4e), reaching $75 \%$. This value is surprisingly close to the mEPSC fraction in recordings from age-matched $b d n f+/+$ hippocampal cultures $(73 \pm 3 \% ; n=19$; data not shown).

\section{The expression of BDNF::EGFP alters dendrite morphology}

It has been suggested repeatedly that dendritic arbors develop under the influence of synaptic input (Van Aelst and Cline, 2004; Dijkhuizen and Ghosh, 2005b). Returning to the shorter expression time of $16 \mathrm{~h}$, we examined the effects of tBDNF on "dendrite elongation" (i.e., the number of dendritic trees exceeding the ROI limits) and "dendrite initiation" (i.e., the number of primary

dendrites per neuron or the number of branch points per ROI). At the time of fixation (DIV6), dendrites could already be distinguished from axons (see Materials and Methods), and previous quantitative analysis of these cultures showed that $>95 \%$ of their combined dendritic length displays immunoreactivity for MAP2 (Salama-Cohen et al., 2006).

Even at first glance, the shape of the transfected neurons hinted at the possibility that tBDNF altered the dendritic pattern of hippocampal neurons, giving the latter a characteristic "bushy" appearance (Fig. 5a). Evaluation of transfection experiments indicates that tBDNF suppressed dendrite elongation while promoting the outgrowth of additional primary dendrites or higher-order branches (Fig. $5 b-d$ ). The effects of high levels of ambient BDNF on the cultures from $b d n f-/-$ mice were weaker but similar (Fig. $5 e-g$ ). The similarity of the responses to exogenous and tBDNF suggests that also in the latter case the structural changes were caused by binding of BDNF to plasma membrane receptors rather than by any kind of intracellular signaling "short-cut." Experiments with neurotrophin receptor blockers confirmed this idea (Fig. 6).

The alteration of dendrite morphology under the influence of BDNF release from transfected neurons in a BDNF-deficient environment either may be caused by an autocrine action or result from a tBDNF-induced change in the synaptic input. To address this issue, we first examined BDNF-expressing neurons for a correlation of the number of VGluT + terminals with dendrite elongation (Fig. 6a) or branch point number (supplemental Fig. $2 b$, available at www.jneurosci.org as supplemental material). Sister cultures from the same experiment were pooled and tested for correlation, as shown in Figure $6 a$. Only cells with more than six VGluT + terminals per ROI were included. In four of five experiments, a significant negative correlation was present between the number of VGluT + terminals and the number of dendritic trees $>50 \mu \mathrm{m}$.

To apply a second and more specific test for the possibility that changes in dendrite morphology were indirect consequences of enhanced glutamate release, we examined the effects of GluR blockers. GluR block produced an additional increase in the glutamatergic synaptic terminal numbers (Fig. $6 d$ ) but prevented the tBDNF-induced decrease in dendrite length (Fig. 6e). It also abolished the negative correlation between dendritic length and the number of VGLuT + terminals (data not shown). The dependency of dendritic branching on GluR activity was less pronounced (Fig. 6f).

That the tBDNF-induced changes in dendrite elongation and branching were mediated by different mechanisms was also shown by blocking TrkB (Fig. $6 b, c$ ). The results with the antibodies against TrkB and $\mathrm{p} 75^{\mathrm{NTR}}$ pointed in the same direction (supplemental Fig. $2 c-f$, available at www.jneurosci.org as supplemental material). Together, these results led to the conclusion that the control of dendrite elongation and initiation displays differential dependence on glutamatergic synaptic activity and relies on different neurotrophin signaling pathways.

\section{Subcellular distribution of tBDNF}

Accumulation of vesicular tBDNF at postsynaptic sites opposite glutamatergic synaptic terminals has been proposed to be a prerequisite for the local regulation of glutamatergic synaptic input (Dijkhuizen and Ghosh, 2005b). The closeness of tBDNF clusters to excitatory synaptic terminals may also contribute to the TrkBand GluR-mediated suppression of dendrite elongation in neurons expressing BDNF. In contrast, a diffuse distribution of tBDNF would be expected to underlie nonregulated release of 
BDNF and a more widespread activation of $\mathrm{p}^{\mathrm{NTR}}$. To obtain more detailed information on the distribution of tBDNF under the given experimental conditions, we applied high-power $(100 \times)$ quantitative fluorescence microscopy to BDNFtransfected dendrites immunostained with an antibody against MAP2, in addition to VGluT staining. This approach revealed that, despite the short expression times, tBDNF formed clusters in otherwise smooth and cylindrical dendrites. A clustered pattern was found in $\sim 50 \%$ of the cells $(n=50)$, typically on the background of a diffuse BDNF::EGFP signal. The remaining cells showed diffuse staining but no clusters. The clusters appeared in a relative uniform size of $\sim 0.25 \mu \mathrm{m}^{2}$ (Fig. $7 a, b)$. In addition to these microclusters, we also observed macroclusters of variable shape, which could be derived either from an accumulation of microclusters or from blobs of intense diffuse staining in first- or second-order branches.

To clarify whether the postsynaptic area opposite a VGluT + terminal is indeed a preferred site of BDNF clusters, we performed dual line plots along thin dendrites contacted by one VGluT + terminal at the side of the dendrite (Fig. $7 b, c$ ). As shown in the specimen of Figure $7 b$, most contacts were faced by more than one BDNF cluster. The average number was $2.4 \pm 0.28(n=15)$. Along a virtual line beside the postsynaptic dendrite, where 0 $\mu \mathrm{m}$ would correspond to the projection of the center of the presynaptic VGluT+ spot, the nearest BDNF cluster was at a distance $\left(x_{1}\right)$ of $0.44 \pm 0.25 \mu \mathrm{m}$; the second closest $\left(x_{2}\right)$ was at a distance of $1.76 \pm$ $0.36 \mu \mathrm{m}$. Dual line plots from 15 boutons (from 7 different neurons and 2 cultures) were normalized to the respective peak values and averaged, as shown in Figure $7 c$. This analysis shows that the region opposite glutamatergic synaptic terminals is indeed a preferred position of BDNF clusters. In neurons that had a clustered distribution of BDNF::EGFP, 75\% of the dendritic sites contacted by a VGluT + terminal $(n=70)$ contained at least one cluster of BDNF::EGFP. These results are consistent with the hypothesis that tBDNF can be released in the immediate vicinity of glutamatergic synaptic terminals.

\section{Discussion}

A defined source of BDNF was created by transfecting neurons from $b d n f-/-$ mice in hippocampal cultures. We report the following new results. (1) Neurons expressing BDNF::EGFP attracted a larger number of synaptic terminals. (2) Postsynaptic BDNF produced a stronger upregulation of synaptic terminal numbers than ambient BDNF. (3) Glutamatergic and GABAergic synaptic terminals reacted in a differential manner to postsynap-
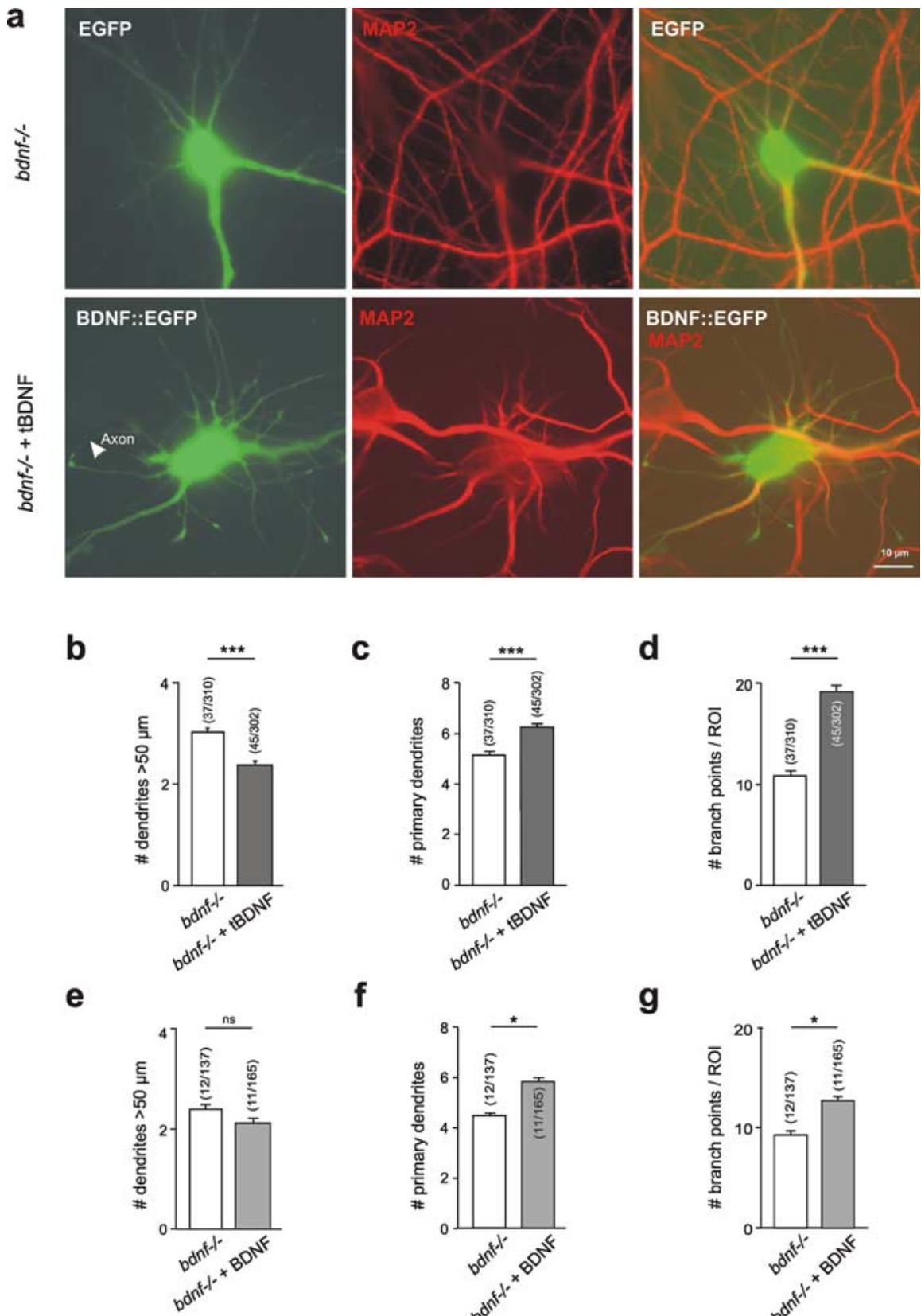

f

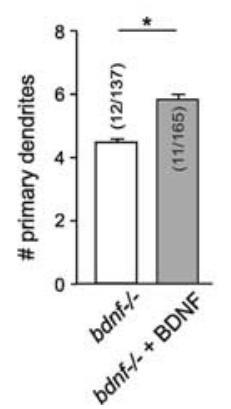

g

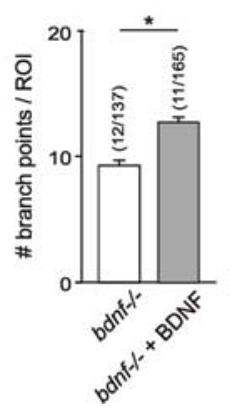

Figure 5. Altered dendritic morphology after BDNF expression in solitary hippocampal neurons in bdnf-/-cultures at DIV6. Expression time was 16 h. $\boldsymbol{a}$, Immunofluorescence images of transfected neurons after immunostaining with antibody against EGFP (green) and MAP2 (Red). In the BDNF-transfected neuron, all neurites except one (arrowhead) were MAP2 positive. $\boldsymbol{b}-\boldsymbol{d}$, Quantification of results with BDNF transfection. $\boldsymbol{e}-\boldsymbol{g}$, Quantification of results from experiments with addition of exogenous BDNF (50 or $100 \mathrm{ng}$ for $16 \mathrm{~h}$ ). ${ }^{* * *} p<0.001$.

tic BDNF, i.e., the glutamatergic input increased, whereas the GABAergic input decreased. (4) BDNF::EGFP-expressing neurons differed from BDNF-deficient neurons in their dendrite morphology: they exhibited weaker dendrite elongation and stronger dendrite initiation. (5) The upregulation of glutamatergic synaptic input and the BDNF-induced downregulation of GABAergic terminal numbers by postsynaptic BDNF required TrkB activity. (6) The suppression of dendrite elongation was also prevented by block of TrkB but required, in addition, GluR activity. (7) Dendritic length decreased with the number of glutamatergic contacts. (8) Application of a function-blocking antibody against $\mathrm{p} 75^{\mathrm{NTR}}$ failed to prevent the changes in synapse numbers and dendrite length but attenuated dendritic branch- 
a

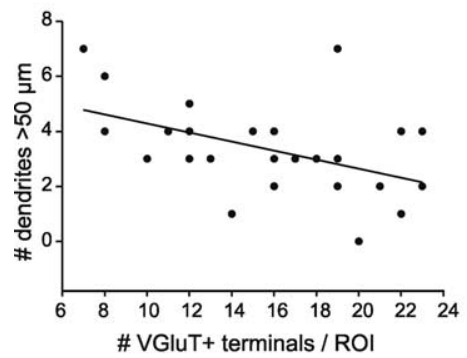

d

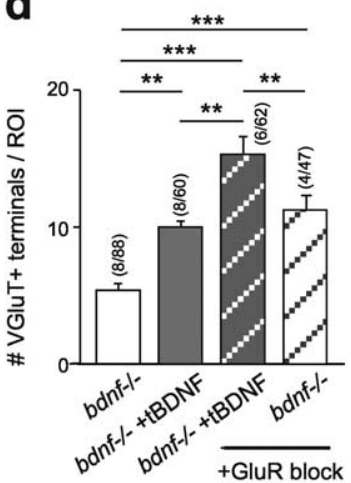

b

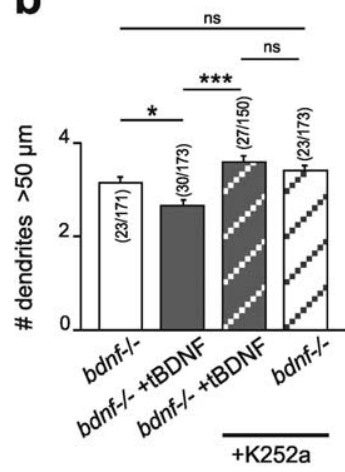

C

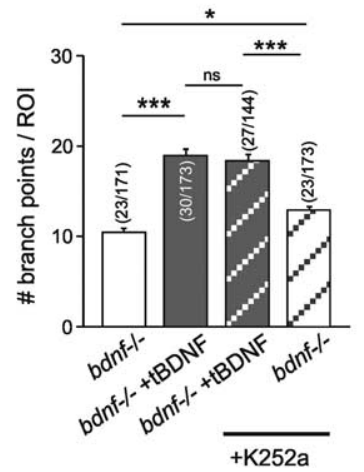

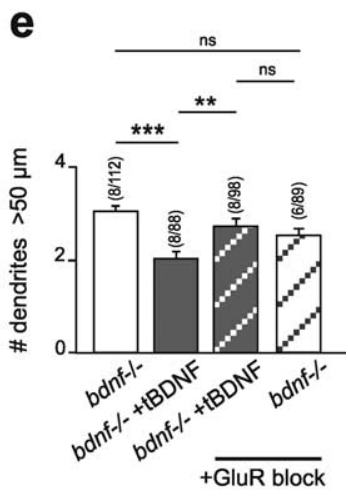

f

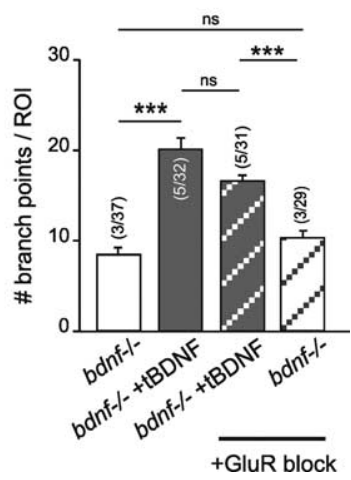

Figure 6. Differential regulation of dendrite elongation and branching by GluR activity and block of TrkB signaling. $\boldsymbol{a}$, Negative correlation between number of VGluT+ terminals and number of dendritic trees exceeding the ROI. This correlation is significant at $p<0.01$. All data points are from one experiment. Similar results were obtained in a total of four experiments. $\boldsymbol{b}$, c, Effects of the tyrosine kinase inhibitor K252a ( $200 \mathrm{~nm}$ ). $\boldsymbol{d}$, Addition of GluR blockers DNQX (20 $\mu \mathrm{M})$, MK801 (1 $\mu \mathrm{M})$, and (RS)- $\alpha$-methyl-4-carboxyphenylglycine $(200 \mu \mathrm{M})$ during the $16 \mathrm{~h}$ expression time of EGFP or BDNF::EGFP does not prevent the tBDNF-induced upregulation of VGluT + terminal number but does produce an additional increase. $\boldsymbol{e}, \boldsymbol{f}$, GluR silencing prevents the tBDNF-induced suppression of dendrite elongation but only weakly counteracts, if at all, the tBDNF effect on dendrite branching. ${ }^{*} p<0.05 ;{ }^{* *} p<0.01 ;{ }^{* * *} p<0.001$. ns, Not significant.

ing. On the basis of these results and previous findings from other laboratories, we discuss the mechanisms of the retrograde and autocrine actions of BDNF as well as a possible indirect route of BDNF action via GluR activity.

TrkB-mediated upregulation of glutamatergic synaptic inputs is induced by postsynaptic BDNF

On the basis of experiments with long-term BDNF treatment or comparison of wild-type and BDNF-deficient hippocampal neurons, previous studies have firmly established the concept that

BDNF potentiates glutamatergic synaptic transmission (Kang and Schuman, 1995; Korte et al., 1995; Figurov et al., 1996). The increased strength of excitatory synapses manifested itself, on the presynaptic side, by increased amplitudes of evoked EPSCs and a decreased paired-pulse ratio (Sherwood and Lo, 1999), an increased frequency of mEPSCs, associated with a larger spine density (Tyler and Pozzo-Miller, 2003; Alonso et al., 2004), an increase in the number of docked vesicles (Tartaglia et al., 2001; Tyler and Pozzo-Miller, 2001), and higher levels of the synaptic markers synaptophysin, synaptobrevin, and synaptotagmin (Tartaglia et al., 2001). On the postsynaptic side, it was found that chronic BDNF treatment increased the size of postsynaptic densities (Collin et al., 2001).

A long-standing question, however, was whether BDNF exerted its stimulatory effect by a retrograde or an anterograde mechanism (Von Bartheld et al., 2001; Howe and Mobley, 2005; Zweifel et al., 2005). A recent study with TrkB knock-out (Luikart et al., 2005) shed light on this issue by showing that selective knock-out of presynaptic TrkB receptors reduced the number of Syp + terminals, whereas postsynaptic BDNF deficiency diminished the number of spines, the size of the postsynaptic densities, and the amplitude of glutamate-induced currents. Our results complement this concept by showing that effects attributed to presynaptic TrkB receptors were induced by a postsynaptic source of BDNF.

Glutamatergic synaptogenesis may also be facilitated by the ability of BDNF-releasing neurons to provide a chemoattractant signal to approaching growth cones (Song et al., 1997; Li et al., 2005); however, BDNF-induced growth cone turning required activity of $\mathrm{p} 75^{\mathrm{NTR}}$ (Gehler et al., 2004), whereas the increase in VGluT + terminal numbers was unaffected by $\mathrm{p} 75^{\text {NTR }}$ blockade. Possibly, in our experiments, the concentrations of released BDNF was too low to activate an axonal growth cone over some distance.

The involvement of $\mathrm{p} 75^{\mathrm{NTR}}$ in the regulation of glutamatergic synapses has been addressed only recently (Woo et al., 2005). This study showed that pro-BDNF, by activating $\mathrm{p} 75^{\mathrm{NTR}}$, facilitates long-term depression of excitatory synapses resulting from a postsynaptic effect, namely reduced expression of the NMDA receptor subunit NR2B. A role of $\mathrm{p} 75^{\mathrm{NTR}}$ in the BDNF-induced stimulation of glutamate release has not been reported.

One should also consider that postsynaptic BDNF may affect the stabilization of glutamatergic terminals through a postsynaptic TrkB-dependent mechanism. In vivo time-lapse imaging of retinal axons in the Xenopus optic tectum revealed that neutralizing endogenous tectal BDNF appreciably enhanced the elimination of GFP-labeled synaptic terminals (Hu et al., 2005); however, a full understanding of the mechanisms of TrkB-mediated synapse stabilization will require additional experiments.

\section{Downregulation of GABAergic synaptic inputs and arrest of dendrite elongation are indirect consequences of the enhanced glutamatergic input}

BDNF is widely regarded as a general modulator of transmitter release, including the release of GABA. Several groups have reported a BDNF-induced increase in the number or size of L-glutamic acid decarboxylase (GAD)-positive terminals (Marty et al., 2000; Aguado et al., 2003; Palizvan et al., 2004), the intensity of GAD65 immunoreactivity (Henneberger et al., 2005), or the frequency of GABAergic mIPSC activity (Palizvan et al., 2004; Baldelli et al., 2005). At least two complexities in the response of inhibitory synaptic terminals may determine the final outcome: (1) elevated expression of GAD65 may be associated with a re- 
duced expression of VIAAT (Henneberger et al., 2005), and (2) the number of GABAergic synaptic terminals and GABA release may depend not only on the degree of presynaptic TrkB activation (where BDNF is supposed to stimulate GABA release) but also on the glutamatergic synaptic activity of the postsynaptic neuron.

A downregulation of VIAAT by BDNF is supported by the present experiments in which (1) VIAAT mRNA levels increased in the chronic absence of BDNF and (2) added BDNF reduced VIAAT levels even below the level in wild-type cultures. The generally reported upregulation of GAD65 in GABAergic synaptic terminals may be caused by the activation of a compensatory mechanism, but from a functional viewpoint, this compensation appears to be insufficient, because the fraction of MIPSCs was lower in neurons expressing BDNF.

Whether BDNF can suppress the formation or stabilization of synaptic terminals may also depend on the strength of depolarizing synaptic input and the degree of maturation of the postsynaptic cell. That strong glutamatergic synaptic input can suppress GABA release via postsynaptic group I mGluRs and the presynaptic cannabinoid $\mathrm{CB} 1$ receptor has already been described for hippocampal neurons (Chevaleyre and Castillo, 2003). Depolarization and $\mathrm{Ca}^{2+}$ influx may also be produced by GABA. Experiments in our own laboratory (J. Akyeli, J. C. Meier, and R. Grantyn, unpublished observations) revealed that the chronic absence of BDNF delays the onset of GABA action by several days, whereas acute addition of BDNF to $b d n f-/-$ neurons abolishes the depolarizing GABA effects within $<1 \mathrm{~h}$, and this was accompanied by a redistribution of immunolabeled KCC2. Thus, in any given preparation, the final outcome of postsynaptic BDNF on GABAergic synaptogenesis will probably depend on the degree of plasticity of the $\mathrm{Cl}^{-}$extrusion system (Rivera et al., 2004). More work is still necessary to clarify the role of BDNF in the postsynaptic differentiation of developing GABAergic synapses.

With regard to the GluR dependency of the BDNF-induced suppression of dendrite elongation, our results are in line with previous results (Lipton and Kater, 1989). Vital imaging studies revealed a suppressive effect of synaptic activity or mGluR ligands on the length of dendritic filopodia (Portera-Cailliau et al., 2003; Lohmann et al., 2005), but the elongation of terminal and nonterminal dendritic branches might be regulated differentially.

\section{Dendritic branching is controlled by $\mathrm{p} 75^{\mathrm{NTR}}$ and glutamatergic synaptic activity}

The experiments revealed a robust effect of both added and tBDNF on dendritic branching. This result is in accordance with a larger number of previous observations with $b d n f$ overexpression (Horch et al., 1999; Yacoubian and Lo, 2000) or the addition of exogenous BDNF (McAllister et al., 1997; Gascon et al., 2005). Furthermore, it has been suggested that an increase in BDNF
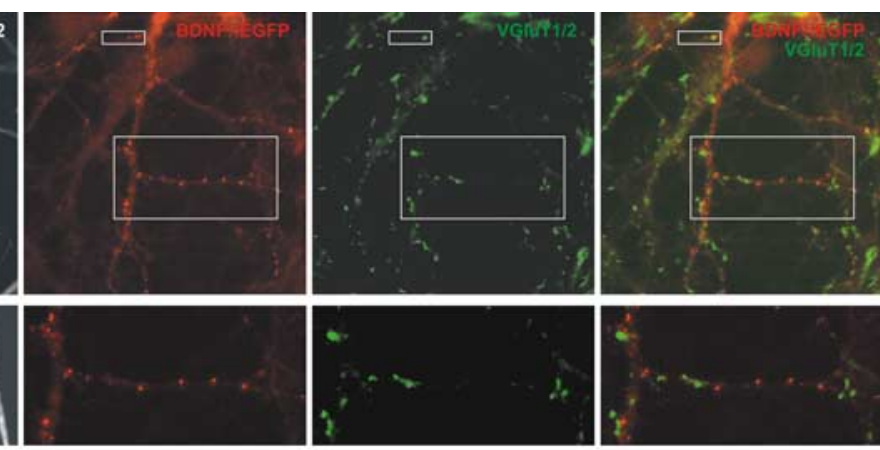

C
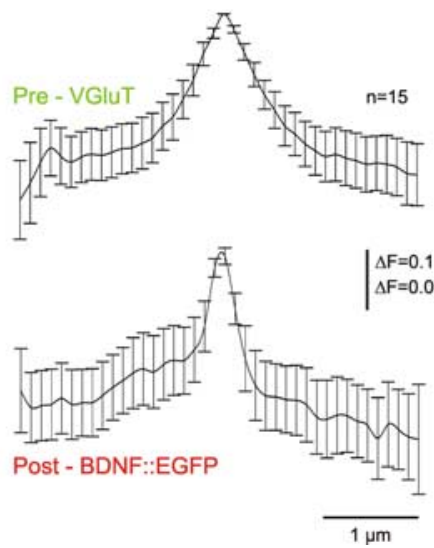

$1 \mu \mathrm{m}$

Figure 7. Clustering of BDNF::EGFP opposite glutamatergic synaptic terminals. $\boldsymbol{a}$, Immunofluorescent images showing the 列 presynaptic plot. The distances of the postsynaptic BDNF fluorescence peaks $x_{1}$ and $x_{2}$ were determined as illustrated in $\boldsymbol{b}$. The averaged postsynaptic line plots show that BDNF preferentially accumulated just opposite the glutamatergic synaptic terminals.

release from transfected neurons could enhance branching in nontransfected close neighbors (Horch and Katz, 2002).

A role of $\mathrm{p} 75^{\mathrm{NTR}}$ in the regulation of dendritic branching was shown recently in hippocampal neurons (Hartmann et al., 2004; Zagrebelsky et al., 2005; Salama-Cohen et al., 2006). These studies suggest that, in general, $\mathrm{p} 75^{\mathrm{NTR}}$ functions as a negative modulator of dendritic branching and spine density. In the context of these findings, one has to assume that the presently observed p $75^{\mathrm{NTR}}$-dependent enhancement of branching by tBDNF is derived from a yet unknown mechanism of interference of BDNF with this action.

That the addition of K252a was ineffective is somewhat surprising in view of the K252a sensitivity of dendrite initiation in cortical explants (Horch et al., 1999) and recent results from pharmacological and molecular perturbation experiments in dissociated cortical neurons (Dijkhuizen and Ghosh, 2005a). These experiments showed that the stimulation of dendrite initiation by exogenous BDNF required activation of mitogen-activated protein kinase and phosphoinositide-3 kinase, i.e., signaling via TrkB. It is possible that the lack of an anterograde source of BDNF in our experimental setting accounts for this discrepancy. The effect may also depend on the density and maturity of the cultures. In younger neurons, with fewer glutamatergic contacts, dendrite initiation might occur in a more autonomous manner under the influence of ambient BDNF acting on p $75^{\text {NTR }}$, whereas 
in older cultures, BDNF may act in a more activity-dependent manner and GluR activation may gain influence also on dendrite branching. However, despite the obvious immaturity of the neurons in the present cultures, GluR block partially reversed the effects of transfected BDNF on the number of branch points.

\section{General conclusions}

Considering that BDNF expression and the availability of neurotrophin receptors, in their turn, are also dependent on neuronal activity, the emergent strong potentiation of excitatory synaptic connections with BDNF-releasing neurons may transiently perturb the homeostatic regulation of developing neuronal networks, and this perturbation could gain further strength by concomitant suppression of GABAergic synaptic input.

\section{References}

Aguado F, Carmona MA, Pozas E, Aguilo A, Martinez-Guijarro FJ, Alcantara S, Borrell V, Yuste R, Ibanez CF, Soriano E (2003) BDNF regulates spontaneous correlated activity at early developmental stages by increasing synaptogenesis and expression of the $\mathrm{K}(+) / \mathrm{Cl}(-)$ cotransporter KCC2. Development 130:1267-1280.

Alonso M, Medina JH, Pozzo-Miller L (2004) ERK1/2 activation is necessary for BDNF to increase dendritic spine density in hippocampal CA1 pyramidal neurons. Learn Mem 11:172-178.

Baldelli P, Hernandez-Guijo JM, Carabelli V, Carbone E (2005) Brainderived neurotrophic factor enhances GABA release probability and nonuniform distribution of $\mathrm{N}$ - and $\mathrm{P} / \mathrm{Q}$-type channels on release sites of hippocampal inhibitory synapses. J Neurosci 25:3358-3368.

Balkowiec A, Kunze DL, Katz DM (2000) Brain-derived neurotrophic factor acutely inhibits AMPA-mediated currents in developing sensory relay neurons. J Neurosci 20:1904-1911.

Bi G, Poo MM (2001) Synaptic modification by correlated activity: Hebb's postulate revisited. Annu Rev Neurosci 24:139-166.

Bibel M, Barde YA (2000) Neurotrophins: key regulators of cell fate and cell shape in the vertebrate nervous system. Genes Dev 14:2919-2937.

Brünig I, Penschuck S, Berninger B, Benson J, Fritschy JM (2001) BDNF reduces miniature inhibitory postsynaptic currents by rapid downregulation of $\operatorname{GABA}(\mathrm{A})$ receptor surface expression. Eur J Neurosci 13:1320-1328.

Chao MV (2003) Neurotrophins and their receptors: a convergence point for many signaling pathways. Nat Rev Neurosci 4:299-309.

Chen ZY, Ieraci A, Teng H, Dall H, Meng CX, Herrera DG, Nykjaer A, Hempstead BL, Lee FS (2005) Sortilin controls intracellular sorting of brainderived neurotrophic factor to the regulated secretory pathway. J Neurosci 25:6156-6166.

Chevaleyre V, Castillo PE (2003) Heterosynaptic LTD of hippocampal GABAergic synapses: a novel role of endocannabinoids in regulating excitability. Neuron 38:461-472.

Collin C, Vicario-Abejon C, Rubio ME, Wenthold RJ, McKay RD, Segal M (2001) Neurotrophins act at presynaptic terminals to activate synapses among cultured hippocampal neurons. Eur J Neurosci 13:1273-1282.

Dijkhuizen PA, Ghosh A (2005a) BDNF regulates primary dendrite formation in cortical neurons via the PI3-kinase and MAP kinase signaling pathways. J Neurobiol 62:278-288.

Dijkhuizen PA, Ghosh A (2005b) Regulation of dendritic growth by calcium and neurotrophin signaling. Prog Brain Res 147:17-27.

Egan MF, Kojima M, Callicott JH, Goldberg TE, Kolachana BS, Bertolino A, Zaitsev E, Gold B, Goldman D, Dean M, Lu B, Weinberger DR (2003) The BDNF val66met polymorphism affects activity-dependent secretion of BDNF and human memory and hippocampal function. Cell 112:257-269.

Ernfors P, Lee K-F, Jaenisch R (1994) Mice lacking brain-derived neurotrophic factor develop with sensory deficits. Nature 368:147-150.

Figurov A, Pozzo-Miller LD, Olafsson P, Wang T, Lu B (1996) Regulation of synaptic responses to high-frequency stimulation and LTP by neurotrophins in the hippocampus. Nature 381:706-709.

Gascon E, Vutskits L, Zhang H, Barral-Moran MJ, Kiss PJ, Mas C, Kiss JZ (2005) Sequential activation of p75 and TrkB is involved in dendritic development of subventricular zone-derived neuronal progenitors in vitro. Eur J Neurosci 21:69-80.
Gehler S, Gallo G, Veien E, Letourneau PC (2004) p75 neurotrophin receptor signaling regulates growth cone filopodial dynamics through modulating RhoA activity. J Neurosci 24:4363-4372.

Goslin K, Asmussen H, Banker G (1998) Rat hippocampal neurons in lowdensity culture. In: Culturing nerve cells (Banker G, Goslin K, eds), pp 339-370. Cambridge, MA: MIT.

Hartmann M, Heumann R, Lessmann V (2001) Synaptic secretion of BDNF after high-frequency stimulation of glutamatergic synapses. EMBO J 20:5887-5897.

Hartmann M, Brigadski T, Erdmann KS, Holtmann B, Sendtner M, Narz F, Lessmann V (2004) Truncated TrkB receptor-induced outgrowth of dendritic filopodia involves the p75 neurotrophin receptor. J Cell Sci 117:5803-5814

Haubensak W, Narz F, Heumann R, Lessmann V (1998) BDNF-GFP containing secretory granules are localized in the vicinity of synaptic junctions of cultured cortical neurons. J Cell Sci 111:1483-1493.

Henneberger C, Grantyn R, Rothe T (2000) Rapid genotyping of newborn gene mutant mice. J Neurosci Methods 100:123-126.

Henneberger C, Kirischuk S, Grantyn R (2005) Brain-derived neurotrophic factor modulates GABAergic synaptic transmission by enhancing presynaptic glutamic acid decarboxylase 65 levels, promoting asynchronous release and reducing the number of activated postsynaptic receptors. Neuroscience 135:749-763.

Horch HW, Katz LC (2002) BDNF release from single cells elicits local dendritic growth in nearby neurons. Nat Neurosci 5:1177-1184.

Horch HW, Krüttgen A, Portbury SD, Katz LC (1999) Destabilization of cortical dendrites and spines by BDNF. Neuron 23:353-364.

Howe CL, Mobley WC (2005) Long-distance retrograde neurotrophic signaling. Curr Opin Neurobiol 15:40-48.

Hu B, Nikolakopoulou AM, Cohen-Cory S (2005) BDNF stabilizes synapses and maintains the structural complexity of optic axons in vivo. Development 132:4285-4298.

Huber LJ, Chao MV (1995) A potential interaction of p75 and trkA NGF receptors revealed by affinity crosslinking and immunoprecipitation. J Neurosci Res 40:557-563.

Kalb R (2005) The protean actions of neurotrophins and their receptors on the life and death of neurons. Trends Neurosci 28:5-11.

Kang H, Schuman EM (1995) Long-lasting neurotrophin-induced enhancement of synaptic transmission in the adult hippocampus. Science 267:1658-1662.

Korte M, Carroll P, Wolf E, Brem G, Thoenen H, Bonhoeffer T (1995) Hippocampal long-term potentiation is impaired in mice lacking brainderived neurotrophic factor. Proc Natl Acad Sci USA 92:8856-8860.

Li Y, Jia YC, Cui K, Li N, Zheng ZY, Wang YZ, Yuan XB (2005) Essential role of TRPC channels in the guidance of nerve growth cones by brain-derived neurotrophic factor. Nature 434:894-898.

Lipton SA, Kater SB (1989) Neurotransmitter regulation of neural outgrowth, plasticity and survival. Trends Neurosci 12:265-270.

Lohmann C, Finski A, Bonhoeffer T (2005) Local calcium transients regulate the spontaneous motility of dendritic filopodia. Nat Neurosci 8:305-312.

Lou H, Kim SK, Zaitsev E, Snell CR, Lu B, Loh YP (2005) Sorting and activity-dependent secretion of BDNF require interaction of a specific motif with the sorting receptor carboxypeptidase e. Neuron 45:245-255.

Lu B (2003) BDNF and activity-dependent synaptic modulation. Learn Mem 10:86-98.

Luikart BW, Nef S, Virmani T, Lush ME, Liu Y, Kavalali ET, Parada LF (2005) TrkB has a cell-autonomous role in the establishment of hippocampal Schaffer collateral synapses. J Neurosci 25:3774-3786.

Marty S, Wehrlé R, Sotelo C (2000) Neuronal activity and brain-derived neurotrophic factor regulate the density of inhibitory synapses in organotypic slice cultures of postnatal hippocampus. J Neurosci 20:8087-8095.

McAllister AK, Katz LC, Lo DC (1997) Opposing roles for endogenous $\mathrm{BDNF}$ and NT-3 in regulating cortical dendritic growth. Neuron $18: 767-778$.

Meier J, Grantyn R (2004) A gephyrin-related mechanism restraining glycine receptor anchoring at GABAergic synapses. J Neurosci 24:1398-1405.

Palizvan MR, Sohya K, Kohara K, Maruyama A, Yasuda H, Kimura F, Tsumoto T (2004) Brain-derived neurotrophic factor increases inhibitory synapses, revealed in solitary neurons cultured from rat visual cortex. Neuroscience 126:955-966. 
Portera-Cailliau C, Pan DT, Yuste R (2003) Activity-regulated dynamic behavior of early dendritic protrusions: evidence for different types of dendritic filopodia. J Neurosci 23:7129-7142.

Pozzo-Miller LD, Gottschalk W, Zhang L, McDermott K, Du J, Gopalakrishnan R, Oho C, Sheng Z-H, Lu B (1999) Impairments in high-frequency transmission, synaptic vesicle docking, and synaptic protein distribution in the hippocampus of BDNF knock-out mice. J Neurosci 19:4972-4983.

Rind HB, Butowt R, Von Bartheld CS (2005) Synaptic targeting of retrogradely transported trophic factors in motoneurons: comparison of glial cell line-derived neurotrophic factor, brain-derived neurotrophic factor, and cardiotrophin-1 with tetanus toxin. J Neurosci 25:539-549.

Rivera C, Voipio J, Thomas-Crussells J, Li H, Emri Z, Sipila S, Payne JA, Minichiello L, Saarma M, Kaila K (2004) Mechanism of activitydependent downregulation of the neurons-specific $\mathrm{K}-\mathrm{Cl}$ cotransporter KCC2. J Neurosci 24:4683-4691.

Salama-Cohen P, Arevalo MA, Meier J, Grantyn R, Rodríguez-Tébar A (2005) NGF and other p75NTR ligands control dendrite morphology in hippocampal neurons by modulating the cellular targets of Notch. Mol Biol Cell 16:339-347.

Salama-Cohen P, Arevalo MA, Grantyn R, Rodríguez-Tébar A (2006) Notch and NGF/p75 ${ }^{\text {NTR }}$ control dendrite morphology and the balance of excitatory/inhibitory synaptic input to hippocampal neurons through Neurogenin 3. J Neurochem 97:1269-1278.

Sherwood NT, Lo DC (1999) Long-term enhancement of central synaptic transmission by chronic brain-derived neurotrophic factor treatment. J Neurosci 19:7025-7036.

Song HJ, Ming GL, Poo MM (1997) cAMP-induced switching in turning direction of nerve growth cones. Nature 388:275-279.

Tartaglia N, Du J, Tyler WJ, Neale E, Pozzo-Miller L, Lu B (2001) Protein synthesis-dependent and -independent regulation of hippocampal synapses by brain-derived neurotrophic factor. J Biol Chem 276:37585-37593.
Teng KK, Hempstead BL (2004) Neurotrophins and their receptors: signaling trios in complex biological systems. Cell Mol Life Sci 61:35-48.

Tyler WJ, Pozzo-Miller LD (2001) BDNF enhances quantal neurotransmitter release and increases the number of docked vesicles at the active zones of hippocampal excitatory synapses. J Neurosci 21:4249-4258.

Tyler WJ, Pozzo-Miller L (2003) Miniature synaptic transmission and BDNF modulate dendritic spine growth and form in rat CA1 neurones. J Physiol (Lond) 553:497-509.

Van Aelst L, Cline HT (2004) Rho GTPases and activity-dependent dendrite development. Curr Opin Neurobiol 14:297-304.

Vicario-Abejon C, Owens D, McKay R, Segal M (2002) Role of neurotrophins in central synapse formation and stabilization. Nat Rev Neurosci 3:965-974.

Von Bartheld CS, Wang X, Butowt R (2001) Anterograde axonal transport, transcytosis, and recycling of neurotrophic factors: the concept of trophic currencies in neural networks. Mol Neurobiol 24:1-28.

Wierenga CJ, Ibata K, Turrigiano GG (2005) Postsynaptic expression of homeostatic plasticity at neocortical synapses. J Neurosci 25:2895-2905.

Woo NH, Teng HK, Siao CJ, Chiaruttini C, Pang PT, Milner TA, Hempstead BL, Lu B (2005) Activation of p75(NTR) by proBDNF facilitates hippocampal long-term depression. Nat Neurosci 8:1069-1077.

Yacoubian TA, Lo DC (2000) Truncated and full-length TrkB receptors regulate distinct modes of dendritic growth. Nat Neurosci 3:342-349.

Yang XL, Xiong WC, Mei L (2004) Lipid rafts in neuregulin signaling at synapses. Life Sci 75:2495-2504.

Zagrebelsky M, Holz A, Dechant G, Barde YA, Bonhoeffer T, Korte M (2005) The p75 neurotrophin receptor negatively modulates dendrite complexity and spine density in hippocampal neurons. J Neurosci 25:9989-9999.

Zweifel LS, Kuruvilla R, Ginty DD (2005) Functions and mechanisms of retrograde neurotrophin signaling. Nat Rev Neurosci 6:615-625. 